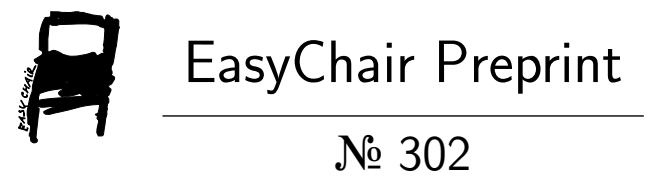

Introduction to Development of Software Support for Training and Testing IT Administrators

Petra Holbíková, Lukáš Králík, Petr Žáček and Roman Jašek

EasyChair preprints are intended for rapid dissemination of research results and are integrated with the rest of EasyChair. 


\title{
Introduction to Development of Software Support for Training and Testing IT Administrators
}

\author{
Petra Holbíková, Lukáš Králík, Petr Žáček, Roman Jasek \\ Department of Artificial Intelligence and Informatics \\ Faculty of Applied Informatics, Tomas Bata University in Zlin \\ Zlin, Czech Republic \\ e-mail: \{holbikova, kralik,zacek,jasek\}@fai.utb.cz
}

\begin{abstract}
The paper is divided into two parts. The first part described used technologies, programming languages, and frameworks used for developing the application Software Support for Training and Testing of IT. The second part is focused on describing the system itself. Mainly are described frontend and backend parts. (Abstract)
\end{abstract}

Keywords—web, learning application development, content management system plug-ins, javaScript frameworks

\section{INTRODUCTION}

People nowadays use the Internet possibly to everything. Entertainment, business, shopping, and also as an effective tool for education are only small part of internet usage. One of the outcomes of our research is the application for the education of users - software support for training and testing IT administrators. In this web application, we focus on effective teaching IT administrators by combining a visual representation of the network and network devices, that are teaching solving IT problems. The basis of this testing is the formation of possible scenarios of real situations that may occur. All of them IT administrator must resolve.

The quality of the provision or management, information technology and safety can largely affect the functioning, efficiency and business performance. For this reason, we created a number of procedural frameworks and methodologies. In the overwhelming majority of it is a set of concepts and practices that allow better planning, use and improve the usage of information and communication technologies, whether on the part of suppliers of IT services or by the customer.

Used technologies are selected so that they cover the largest proportion of users, that are the multi-platform technologies with responsive design to cover the users with mobile devices and tablets. Due to this form is user education accessible almost anywhere, where the user can connect to the Internet. An application design cares to current standards and userfriendly and intuitive control.

\section{USED TECHNOLOGIES AND BASIC TERMS}

\section{A. PHP: Hypertext Preprocessor}

PHP is a server-side scripting language, which was developed by Rasmus Lerdorf in 1995. It is an Open Source technology, cross-platform, therefore it can run on most Web servers and existing OS. It is used for creating dynamic websites, such as online shops, forums, content management systems, information systems and dynamic presentations. The syntax of the language is based on Perl, C and Java. The current stable release is version 7.1.1 of PHP. Compared to other Web server technologies, PHP is more used, reaching $82.5 \%$, while the second most frequently used technology ASP 15.3\%.

The first version was called PHP/FI and was released in 1995. It contained a simple set of Perl scripts, named Personal Home Page Tool and implementation in language C-Form Interpreter, which could communicate with databases. The following version of PHP/FI 2.0 was released in 1997. This technology benefited over 50,000 domains by that time.

PHP 3 was officially released after nine months of testing in June 1998. The development of the project joins Andi Gutmans and Zeev Suraski, who completely rewrote it and added a huge amount of expansion. Original name Personal Home Page Tools was renamed to PHP: Hypertext Preprocessor. PHP 3 was used for approximately $10 \%$ of all Web servers.

In the next version of PHP 4 was the most significant achievement reprocessing core PHP engine. His innovations increase performance and improve the modularity of PHP's code base. The fourth version of PHP was released in 1999. PHP 4 joined support HTTP sessions, input buffering, more secure processing of inputs and more.

Nowadays we use the latest released version 7, which skipped version 6 , that was never officially released.[1]

\section{B. Hypertext Markup Language}

HyperText Markup Language (HTML) is a markup programming language designed for creating web applications and websites on the World Wide Web. Its development is backward influenced by the development of Web browsers. It is based on the universal markup language SGML and characterized by a plurality of tags and their attributes required structure, which includes the declaration document type definition, root element, the document header and the body.

Language development began in 1989 in CERN. The first unofficial version was developed by Tim Berners-Lee and Robert Caillau. Then this version was linked HTTP. Along with this development was created the first Web browser, called 
WorldWideWeb. The first website, CERN, was launched in 1991. The CERN website was written in version 0.9 and did not support a graphical user interface.

After two years, in 1993, was presented a proposal to HTML version 2.0 and also Mosaic browser came out. It became the first Internet browser that supports graphical interface. Standard HTML 2.0 was released in 1995 and newly included graphics support and forms.

HTML version 3.0 was never for its complexity accepted as a standard. The following HTML 3.2 has been extended to tables, rustic elements, and text alignment. Standard was released by the international community W3C in January 1997.

A new standard HTML 4.0 was released at the end of the same year. In this version have been standardized frames. Newly the appearance is influenced by the connected styles.

This version is followed HTML 4.01. The development of HTML 5 version started in 2007. Finalization of the specifications of this release is estimated for the year 2022.[5]

\section{Cascading Style Sheets}

Cascading Style Sheets(CSS) is a language used to define the appearance of web pages. It is mainly used in link with HTML and XHTML but can be used also in link with XML documents. The development was designed and is supported by the World Wide Web Consortium.

The first version of CCS 1 was released in 1996. Until 2000 only the Opera browser has full support for CSS 1. The second version of CSS 2 was released in May 1998. Its use is not recommended. Instead of this version, it is recommended CSS 2.1, which was standardized in 2011. It can be used CSS version 3 too. In developing is also the CSS 4, which is not supported in any of the browsers yet.[5]

\section{JavaScript}

JavaScript is object-oriented, cross-platform scripting language, created by Brendan Eich. It is used in web technology to operate with graphical user interface iterative elements, animations, and effects. Javascript syntax is similar to language $\mathrm{C} / \mathrm{C}++$ or Java. The development of this language had begun in 1995. Current version EMCAScript 6 was released June 17, 2015.

In our project, we use two common JavaScipr libraries. The first is widely used jQuery library, that is used here for user and application interaction. The use of a library is especially for click, double click or hover actions, loading JSON array, asynchronous data load and more interactions.

D3.js library is used for drawing data on HTML canvas element.

\section{E. WordPress}

WordPress is an open source content management system (CMS). It is Written in PHP and MySQL. According to official statistics, it is used as a CMS for more than $27 \%$ of websites in the world and thus overcomes the open source CMS like Joomla or Drupal, which holds under three percent.[7]

Development of plug-ins and widgets is due to a large community around the system and easily accessible thanks to site architecture easy to understand. Simplified site architecture can be seen in the figure below.

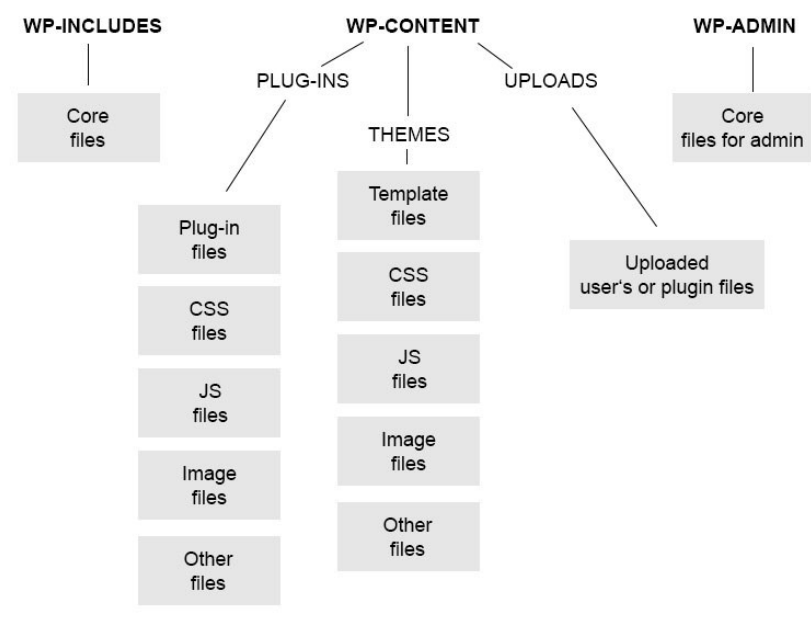

Fig. 1. Simplified WordPress site architecture

The WordPress root directory has three subdirectories, wpadmin, wp-content, wp-includes. Plug-ins or theme developers works only in wp-content directory, where developers upload files with his code. [6]

\section{DEVELOPMENT OF LEARNING SYSTEM}

Software Support for Training and Testing of IT Administrators application is created with web technologies described earlier. These technologies were selected based on the multiplatform and accessibility via the Internet. PHP, MySQL, HTML5, JavaScript and CSS technologies are used for programming a plug-in for WordPress.

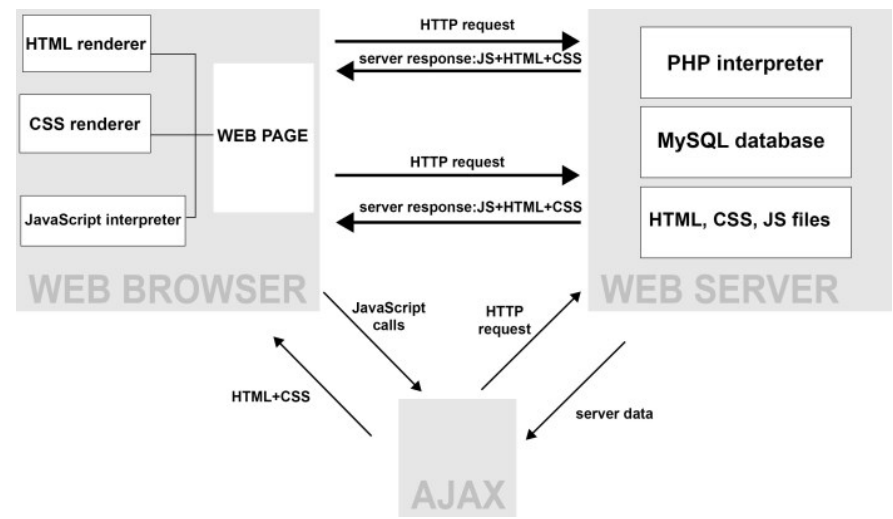

Fig. 2. Comunication between browser and web server

Firstly was made the technical specifications of the project paper, graphic design and the database structure. The application will be divided into backend part, where the user (administrator) sets individual nodes such as a PC, router, or server, and define 
their properties. Also, it is possible to add individual testing tasks and scenarios.

Front-end part is designed for learning and testing users. The user interface is divided into two basic elements, a list of error messages and devices available for solving task or situation. The node with a device is interactive, offers the user possible actions, that can be done with each node, due to the user can resolve the situation. The application will be filled with test data after the backend and frontend programming. The last part of the development of an application is to write documentation and tutorials on how to work with the application. We can achieve quality learning material with visual elements. Now we will focus on describing parts of the frontend and backend.

\section{A. Frontend}

Front-end environment development starts by the design of its functionality. The main requirements are the data visualization and the user interaction with application to educate and testing. For this reason, the chosen form is a network with nodes and their links. We can easily visualize the connections between nodes and their link to each other. Each node represents a device on the network. It carries information about the device and actions, that individual device offers. The use of prepared scenario is for learning and then for testing. We can evaluate whether the user makes the correct steps to resolve the defined problem.

Frontend uses the technology of JavaSqript frameworks. JQuery is used to create a responsive design of HTML canvas element, which can not be created using CSS. Subsequently, we load the JSON file, in which are stored the information needed to generate the graphics of individual nodes. This basic information in the file is divided into the nodes and connectors.

Basic node information is:

- ID

- The size of node

- Image

- Title

- Description

- Actions

In the second group are links, which has an array of the start node ID and ID of an end node. [3,4]

Using the d3.js library are generated graphic elements nodes and their links in canvas. Due to the node information are stored in JSON file, we can generate every time different set of nodes and error list. The limit is only the number of created scenarios.

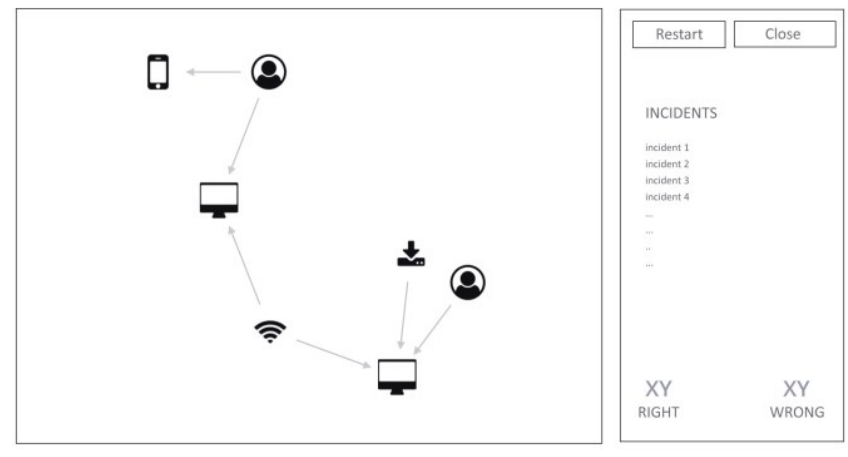

Fig. 3. Frontend application wireframe - default state

On the main screen user can see the network with devices on the left and info part section on the right. This section is changed based on the work with the system. The user sees a list of incidents and a result score on the screen in the default state as it is shown in fig.3.

When is any device (node) clicked the info part section is changed. The device information, description and list of device action is now shown. By the clicking on device action can be network incident or any part of incident fixed. The device info part is shown in fig. 4 .

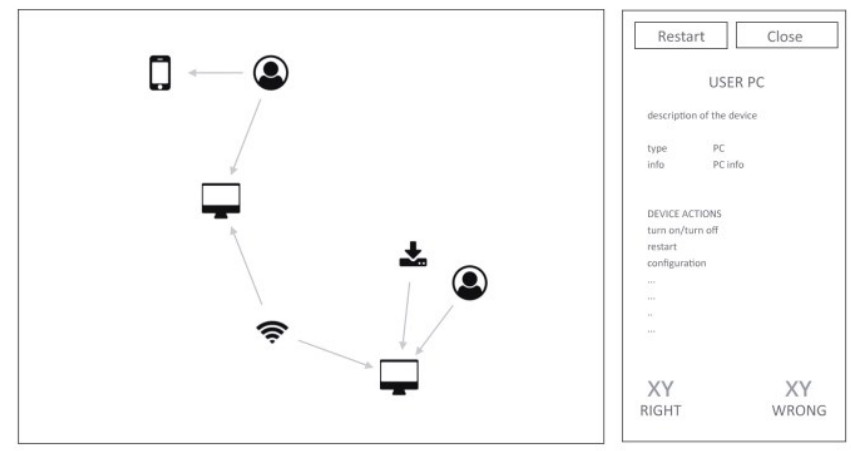

Fig. 4. Frontend application wireframe - device info part

\section{B. Backend}

The creation of backend part can be divided into several parts. The first is to decide which information will be stored for each node. For maximum compatibility was used basic functions of WordPress. We decided to use post types and their metadata. Here we created a new type of post named "Device type". Here administrator inserts all types of devices, which can be found in networks, for example, PC, smartphone, switch, router, flash disc, printer or others. To each new Device type entry is defined several types of metadata; for example, a list of actions that are possible to do with the device, video or audio recording upload, icon or a description of the device.

Another new type of post is called "Device". Within these entries are build individually networks. One new entry represents one node. We must assign a type of this device from the post type described earlier. Also, we can redefine metadata. 
All these records are stored in tables, that works with the core of the system. We do not have to program new functions to obtaining data and will use the basic functions of WordPress.

For adding links between nodes is created a page with the form in which we can find two select boxes with a list of the available devices, in other words, a list of "Device" post type. The administrator can select the start node and end node and save the relationship. The database stores again the metadata, that contains an ID of an end node. The metadata is stored within an entry of a start node device. Thanks to this, we are able to generate a source file for front-end data.[2].

Another part of the backend is storing error devices. For this cases, we created a custom database table, which contains this information:

- ID

- ID of error device

- ID of action to solve the problem

- ID of action, one incident can cause more error devices

The last part is the creation of the list of incidents. For storing incidents we also created a new table in the database with columns:

- ID

- Name of the incident

- The incident info

- The category

- The weight of the incident

Filling up this two tables with error devices and incidents can be easily generated a list for user frontend.

\section{CONCLUSION}

Information technology nowadays completely part of all sectors of human activity and it can be stated that the current business is dependent on information technology.

Software support for training and testing of IT administrators application is based on randomly generated events to test their skills and especially on skills of making decisions in the area of incident management. The whole test takes place in a simple virtual environment, where are the basic elements of the information system and IT infrastructure.

The goal of testing is to make the IT administrator be able to adequately classify individual events on information security incidents and incidents. Based on the classification performed appropriate measures (temporary solution, revealing the problem, identify a possible cyber attack, incident escalation, etc.) and informed the appropriate person.
Development of this software tool will become a unique training tool for improving the quality and effectiveness of IT administrators. The actual application development thanks to widely used CMS Wordpress a well described JavaScript frameworks fast and efficient.

\section{ACKNOWLEDGMENT}

This work was supported by No. IGA/CebiaTech/2017/007 from IGA (Internal Grant Agency) of Tomas Bata University in Zlin..

\section{REFERENCES}

[1] PHP.net. Accessed April 10, 2017. http://php.net/.

[2] Aggrawal, Ritu. 2016. "Developing Website in an Open Source Content Management System (CMS) WordPress." International Journal of Innovations in Engineering and Technology 7 (1).

[3] Amr, Tarek and Rayna Stamboliyska. 2016. Practical D3.Js. Berkeley, CA: Apress

[4] Amr, Tarek and Rayna Stamboliyska. 2016. Practical D3.Js: Master the use of D3.Js in the Real World. New York: Apress.

[5] W3schools.com. Accessed April 10, 2017. https://www.w3schools.com/

[6] WordPress.org. Accessed April 10, 2017. https://wordpress.org/

[7] W3Techs."Web Technology Surveys" Accessed April 10, 2017. https://w3techs.com/technologies/overview/programming_language/all 